\title{
Exclusion of Generalist Pathogens in Multi-host Communities
}

\author{
J. V. Greenman* \\ Department of Computing Science and Mathematics, \\ University of Stirling. \\ Stirling, FK9 4LA, Scotland, UK \\ tel: +44(0)1786-467460; fax: $+44(0) 1786-464551$; \\ email: j.v.greenman@stir.ac.uk
}

\author{
A. S. Hoyle \\ Department of Computing Science and Mathematics, \\ University of Stirling. \\ Stirling, FK9 4LA, Scotland, UK \\ tel: $+44(0) 1786-467467$; fax: $+44(0) 1786-464551$; \\ email: ash@maths.stir.ac.uk
}

(C) 2008 by The University of Chicago.

Accepted for publication by American Naturalist on 23 April 2008.

http://doi.org/10.1086/590967

Keywords: multi-host SIS model, apparent competition, invasion threshold, biological control, force of infection, frequency-dependence.

* Corresponding author 


\begin{abstract}
Knowing how to control a pathogen that infects more than one host species is of increasing importance because the incidence of such infections grows with continuing environmental change. Of concern are infections transmitted from wildlife to humans or livestock. To determine which options are available to control a pathogen in these circumstances, we analyse the pathogen invasion matrix for the multi-host susceptible-infected-susceptible model. We highlight the importance of both community structure and the column sum or row sum index, an indicator of both force of infection and community stability. We derive a set of guidelines for constructing culling strategies and suggest a hybrid strategy that has the advantages of both the bottom-up and the topdown approaches, which we study in some detail. The analysis holds for an arbitrary number of host species, enabling the analysis of large-scale ecological systems and systems with spatial dimensions. We test the robustness of our methods by making two changes in the structure of the underlying dynamic model, adding direct competition and introducing frequency-dependent infection transmission. In particular, we show that the introduction of an additional host can eliminate the pathogen rather than eliminate the resident host. The discussion is illustrated with a reference to bovine tuberculosis.
\end{abstract}




\section{Introduction}

Apparent competition mediated by a pathogen (Holt and Pickering 1985; Hudson and Greenman 1998) is an important aspect of the behaviour of a multi-host shared-pathogen system. Host species act as though they are in competition even if they are not directly competing for resources. Theory implies that the abundance of a species can be considerably reduced, and in some cases excluded, when a second host species acting as a reservoir for the infection invades its habitat (Haydon et al. 2002). Several possible candidates for apparent competition in the wild have been suggested, for example, the exclusion of moose (Alces alces) and caribou (Rangifer tarandus) in Nova Scotia and New Brunswick, Canada, following the invasion of white-tailed deer (Odocoileus virginianus; pathogen, Parelaphostrongylus tenuis; Anderson 1972; Anderson and May 1986) and the decline in the United Kingdom of the gray partridge (Perdix perdix) in the presence of the ringnecked pheasant (Phasianus colchicus; pathogen, Heterakis gallinarum; Tompkins et al. 2001).

Of particular concern are those pathogens that cross from wildlife species to humans or their livestock, threatening public health and economic livelihood. Several such pathogens emerged in the 1960s. The Machupo virus in Bolivia in 1962 was spread by rodents acting as the reservoir host (Johnson et al. 1965; Garrett 1994), invading the territory of the naive and highly vulnerable human population as a result of changes in agricultural practice. For the Ebola virus in Zaire in 1976, fruiteating or insectivorous bats were the most likely reservoir host (Daszak et al. 2000). An important example of livestock affected by an infection that was spread by wildlife in the United Kingdom is that of bovine tuberculosis (TB; causative agent, Mycobacterium bovis). A recent large-scale field trial (the Randomised Badger Culling Trial; Donnelly et al. 2006; Bourne et al. 2007) showed that, although badgers (Meles meles) are the main source of the infection among wildlife (Krebs et al. 1998), culling badgers does not always result in a reduction in bovine TB (Donnelly et al. 2003). This surprising result has been attributed to the disruption of badger communities caused by the culling (Woodroffe et al. 2006).

In complex ecosystems, identifying the main reservoirs of infection can be a formidable challenge. This is illustrated by the continuing search for the source of the Ebola virus (Daszak et al. 2000) and the effort that has been made to unravel the dynamics of rabies epidemics in the Serengeti region of Tanzania (Cleaveland and Dye 1995). Without reliable information, control measures may be ineffective, if not counterproductive.

Culling is one way of trying to control a pathogen, either through direct anthropogenic intervention or through the introduction of direct competitors or predators. For example, the Machupo virus epidemic was ended by the importation of domestic cats to act as predators. Vaccination, which transfers susceptible individuals to an immune class, is a more benign method of control (Roberts 1996). However, vaccines may be unavailable or prohibitively expensive. Another approach is for the authorities to use regulatory controls and demand strict adherence to biosecurity standards to reduce inter- and intra-infection transmission rates (Ferguson et al. 2001). In cases of highly virulent diseases, quarantine may have to be imposed (McLean et al. 2006).

Even if we restrict our attention to the first two non-regulatory methods of control, many issues still remain. Do we need to control all hosts to exclude the pathogen? If not, then which ones need to be controlled? Is it those that exert the largest force of infection, or is some other criterion more appropriate? In this article we address these issues, basing our analysis on the simplest non-trivial 
model available, the multiple-host susceptible-infected-susceptible (SIS) model (Dobson 2004). The key element in the analysis is the invasion matrix J, obtained from the SIS model in the approximation that the infection is rare. The stability properties of this matrix indicate whether the pathogen will invade or be excluded.

Many of the familiar cases of generalist pathogens involve, in effect, only two host species, but there are other cases that involve more (e.g.,West Nile virus; Petersen and Roehrig 2001). The number of such cases is likely to increase with a better understanding of complex ecosystems and with continuing environmental change. Our analysis is, therefore, a general one, looking at multi-host systems of arbitrary size. This gives us the opportunity to model systems with a spatial dimension, where the same host on a different patch is considered to be a different host. This idea has been implemented for the phocine distemper virus affecting harbor seals (Phoca vitulina) in European waters (Swinton et al. 1998; Dobson and Foufopoulos 2001) and can be used to study social perturbation due to badger culling in the case of bovine TB.

After introducing the equations of our model and deriving its pathogen invasion matrix, we keep the mathematical details to a minimum. A more complete discussion can be found in the appendix in the online edition of the American Naturalist.

\section{Fundamentals}

Two host species sharing a pathogen are modelled by the equations

$$
\begin{gathered}
\frac{d H_{i}}{d t}=r_{i}\left(1-\frac{H_{i}}{K_{i}}\right) H_{i}-\alpha_{i} I_{i} \\
\frac{d I_{i}}{d t}=S_{i}\left(\beta_{i i} I_{i}+\beta_{i j} I_{j}\right)-d_{i} I_{i}
\end{gathered}
$$

where $i, j=1,2$ and $i \neq j$, with density-dependent infection transmission (McCallum et al. 2001), no latency, and no natural immunity. Here $S_{i}, l_{i}$, and $H_{i}=S_{i}+l_{i}$ are the ith host susceptible, infectious, and total population densities, respectively. Parameters $a_{i}, b_{i}, \alpha_{i}$, and $\gamma_{i}$ denote per capita birth rate, (additive) natural mortality rate, infection-induced mortality rate, and recovery rate, respectively, for the ith host, with $r_{i}=a_{i}-b_{i}$ and $d_{i}=b_{i}+\gamma_{i}$. Parameter $d_{i}$ measures the rate of population loss from the infectious state and its inverse, the average lifetime of the disease; $K_{i}$ is the ith host carrying capacity, and $\beta_{i j}$ is the rate of infection transmission from host $j$ to host $i$. For the moment, we suppose that the cross-transmission rate $\beta_{i j}$ is nonzero $(i \neq j)$. With no direct competition, the two host species asymptotically tend to their own carrying capacity levels in the absence of the infection when $r_{i}>0$.

In the rare-invader approximation, it is assumed that the number of infectious hosts is initially so small that the total host population is close to its carrying capacity level; that is, $H_{i} \approx S_{i} \approx K_{i}$. The infectious population equation then becomes

$$
\frac{d I_{i}}{d t}=K_{i}\left(\beta_{i i} I_{i}+\beta_{i j} I_{j}\right)-d_{i} I_{i}
$$


where $i, j=1,2$ and $i \neq j$. In vector terms, $d \underline{v} / d t=\mathrm{J} . \underline{v}$, where $\underline{v}=\left(I_{1}, I_{2}\right)^{T}$, with $T$ denoting the transpose operation and where $J$ is the invasion matrix given by equation (2a) in the $2 \times 2$ case and by equation $(2 b)$ in the $3 \times 3$ case,

$$
\begin{aligned}
& \mathbf{J}=\left[\begin{array}{cc}
K_{1} \beta_{11}-d_{1} & K_{1} \beta_{12} \\
K_{2} \beta_{21} & K_{2} \beta_{22}-d_{2}
\end{array}\right] \\
& \mathbf{J}=\left[\begin{array}{ccc}
K_{1} \beta_{11}-d_{1} & K_{1} \beta_{12} & K_{1} \beta_{13} \\
K_{2} \beta_{21} & K_{2} \beta_{22}-d_{2} & K_{2} \beta_{23} \\
K_{3} \beta_{31} & K_{3} \beta_{32} & K_{3} \beta_{33}-d_{3}
\end{array}\right]
\end{aligned}
$$

with obvious generalization for an arbitrary number of host species. There will be pathogen invasion when the leading eigenvalue $\lambda$ of $\mathbf{J}$ is positive, and pathogen exclusion will occur when the leading eigenvalue $\lambda$ of $\mathrm{J}$ is negative. At threshold, $\lambda=0$, and so the determinant of $\mathrm{J}$, det $\mathrm{J}$, is also 0 , being the product of the eigenvalues of J. So, the threshold for invasion lies on the curve (or surface) det $\mathrm{J}=0$.

\section{Control of an Infected Two-Host System}

\section{Control Options}

Our first objective is to determine which options are available for the exclusion of a pathogen that is infecting two host species, as described by model (1). Our primary control will be (partial) culling (i.e., forced increase in natural mortality), but as we will show, the results obtained apply equally to other controls, such as vaccination and restricted access to resources. We generalize the term "culling" to include these other controls. Culling affects only the diagonal elements of the invasion matrix J. For example, if we cull host 1 by $x$, then $b_{1}$ and, hence, $d_{1}$ will increase by $x$. So the $(1,1)$ element of $\mathrm{J}$ in matrix (2a) will decrease by $x$.

The options for control depend on whether the host species is a reservoir host (i.e., a species that alone can support the pathogen). Figure 1 makes this distinction clear. It shows two possible configurations for the pathogen exclusion threshold $\pi$ in the $x-y$ culling plane (where $x$ and $y$ denote the increases in the mortalities of host 1 and host 2, respectively, due to culling).

Configuration $A$, with threshold $T T$ intersecting neither culling axis, corresponds to both hosts being reservoir hosts. Both hosts need to be culled because there are no points in the pathogen exclusion region $E$ where $x=0$ or $y=0$. In configuration $B$, the threshold $T$ intersects the $X$-axis, corresponding to host 2 not being a reservoir host. In this case, pathogen exclusion can be achieved by culling only reservoir host 1 (keeping $y=0$ ). That host 2 need not be culled would be obvious if host 1 were culled to extinction, but the objective is to exclude the pathogen and not the hosts. 

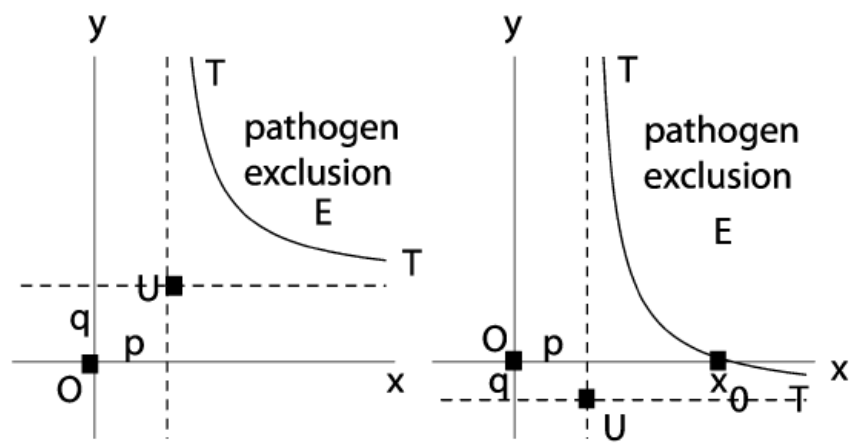

Figure 1: The pathogen invasion/exclusion threshold $\pi$ plotted against the culling of host $1(x)$ and host $2(y)$. Crossing TT from above, the pathogen invades; crossing $T T$ from below, the pathogen is excluded; $T T$ is the upper branch of a hyperbola with asymptotes shown as dashed lines intersecting at centre $U$ with coordinates $(p, q)$. $\ln A$, both hosts are reservoir hosts. In $B$, only host 1 is a reservoir host. The objective of the culling strategy is to reach exclusion region $E$ from the origin $O$.

In case $B$ (fig. $1 B$ ), for single culling, the reservoir host needs to be culled by at least $x_{0}$ to exclude the pathogen (where $x_{0}$ is given by eq. [A2] in the appendix). If culling does not discriminate between susceptible and infectious hosts, then there is the possibility that the reservoir host will also be excluded. To avoid this, the value of $x$ should not exceed the growth rate $r_{1}$. If the limits $x_{0}<x<r_{1}$ cannot be satisfied (e.g., if $r_{1}<x_{0}$ ), then both hosts have to be culled (the hosts sharing the cost of lowered abundance in reducing the reservoir of infection to below critical size; fig. 1B). The problem does not arise if a test (for infection) and cull (if positive) strategy is in place.

There is a third scenario in which neither host species is a reservoir host but together they form a reservoir community (i.e., a community that supports the pathogen). The threshold curve $\pi$ then crosses both axes, and the pathogen can be excluded by culling either one of the two hosts, subject to the proviso that neither host is excluded in the process. This scenario provides an example where a host species has to be culled even though it does not support the pathogen when alone. The connection between the geometry of figure 1 and the algebra of the matrix in equation (2a) can be found in "The Infection Threshold" in the appendix.

\section{Generalized Culling}

Besides direct culling, it is sometimes possible to control a host species by restricting access to resources (e.g., habitat or food), thereby reducing its effective carrying capacity $K$. We will refer to this as $K$-resource control. In "Generalized Culling" in the appendix, we show that, as far as threshold behavior is concerned, reducing $K$ by $\delta K$, for example, is equivalent to culling at a rate

$$
x=\frac{k d}{1-k}
$$


Here, $k \mathrm{p} \mathrm{d} K / K$ is the proportionate reduction in $K$, with $0 \leq k \leq 1$, and $1 / d$ is the average lifetime of the infection.

Control by vaccination can be considered to be an example of $K$-resource control because one can show that vaccination reduces the equilibrium-susceptible population $K$ by a proportionate amount $k=p /[1+(\gamma / b)]$, where $b$ is natural mortality, $p$ is the proportion of the population vaccinated at birth, and $g$ is the rate of loss of immunity. Equation (3) gives the equivalent culling rate. If vaccination does not offer lifelong protection (i.e., $\gamma>0$ ), then vaccination may not be able to reduce the susceptible population sufficiently for pathogen exclusion to occur because $k<1 /[1+$ $(\gamma / b)]<1$.

\section{Controlling Three or More Hosts}

\section{Stability of Host Species and Host Sub-communities}

As we have seen, the distinction between reservoir hosts and non-reservoir hosts (i.e., those not supporting the pathogen) is important. How do we use the invasion matrix to identify which host species is which? We are, for the moment, taking the off-diagonal elements $\left(K_{i} \beta_{i j}\right)$ to be positive (rather than 0 ), but the diagonal elements $\left(K_{i} \beta_{i i}-d_{i}\right)$ can be of either sign. If positive, then the corresponding host, host $i$, is a reservoir host (and if negative, host $i$ is a non-reservoir host), because $K_{i} \beta_{i i}-d_{i}=d_{i}\left(R_{0 i}-1\right)$, where $R_{0 i}$ is the basic reproduction number for host $i$.

To understand the properties of the invasion matrix $\mathrm{J}$, we need to consider the invasion matrices of sub-communities of host species. They can be found straightforwardly from $\mathbf{J}$ by removing all the rows and columns relating to hosts outside the sub-community of interest. For example, consider the invasion matrix J for a four-host community, given as matrix (4a). The invasion matrix for the sub-community without host 1 is given in matrix (4b) as $J_{1}$ and without hosts 1 and 2 in matrix (4c) as $J_{12}$ :

$$
\begin{aligned}
\mathbf{J} & =\left[\begin{array}{cccc}
0.2 & 0.3 & 0.4 & 0.5 \\
0.1 & 0.1 & 0.2 & 0.3 \\
0.2 & 0.4 & -0.2 & 0.2 \\
0.3 & 0.6 & 0.3 & -0.5
\end{array}\right] \\
\mathbf{J}_{\mathbf{1}} & =\left[\begin{array}{ccc}
0.1 & 0.2 & 0.3 \\
0.4 & -0.2 & 0.2 \\
0.6 & 0.3 & -0.5
\end{array}\right] \\
\mathbf{J}_{12} & =\left[\begin{array}{cc}
-0.2 & 0.2 \\
0.3 & -0.5
\end{array}\right]
\end{aligned}
$$

Note that hosts 1 and 2 are reservoir hosts and hosts 3 and 4 are non-reservoir hosts.

For the sake of brevity, we will say that a reservoir Sub-community (one that supports the pathogen) is unstable against pathogen invasion and a non-reservoir sub-community (one that does not support the pathogen) is stable against pathogen invasion. The sub-community of matrix (4c) is stable, whereas those of matrices (4a) and ( $4 b$ ) are unstable, as can be checked by finding their eigenvalues. To help analyse the properties of these sub-communities, there are some general rules that can be applied. The most important of these, to which we will refer several times later in 
"Discussion" as rule 1, is that a stable sub-community cannot contain within it a host or collection of hosts that support the pathogen. So there is no stable sub-community in matrix (4a) that contains hosts 1 or 2. A stable sub-community, therefore, contains only Non-reservoir hosts, but the converse is not necessarily true. If we increase the $(1,2)$ element of matrix $(4 c)$ to 0.4 , we obtain an unstable sub-community containing only non-reservoir hosts. We will call a stable sub-community maximal if when adding any other host to it, it becomes unstable. The only maximal stable sub-community (MSS) of matrix (4a) is the one that consists of hosts 3 and 4 . There would be two single-host MSSs (host 3 and host 4 ) if the $(3,4)$ element of matrix $(4 a)$ were 0.4 .

\section{A Culling Algorithm}

Choose an MSS and add the other host species one by one, sufficiently culling the added host at each stage to render stable the extended community. This will be achieved if the culling, $x$, is greater than $x_{0}=\operatorname{det} \mathbf{J}^{*} / \operatorname{det} \mathbf{J}^{* *}$, where $\mathbf{J}^{*}$ is the invasion matrix for the extended sub-community before culling and $\mathbf{J} * *$ is the invasion matrix of the unextended sub-community (see "The Culling Algorithm" in the appendix for justification). So,

$$
x=\frac{\operatorname{det} \mathbf{J}^{*}}{\operatorname{det} \mathbf{J}^{* *}}+a
$$

where $a$ is any positive number. For example, in invasion matrix (4a), first add host 2 to its MSS as defined by hosts 3 and 4 . Then $\mathbf{J} *$ is given by matrix (4b) and $\mathbf{J} * *$ is given by matrix (4c), and so, by equation (5), host 2 needs to be culled by an amount $x=(0.14 / 0.04)+a=0.35+a$. Culling host 2 by this amount generates a sub-community with an invasion matrix given by matrix (4b) but with $x$ subtracted from its $(1,1)$ element to allow for the culling. This forms the new matrix $\mathbf{J}^{* *}$ when host 1 is added, whereas matrix (4a) with $x$ subtracted from its $(2,2)$ element forms the new matrix $\mathbf{J}^{*}$. So, at the second stage, a pathogen-free community is obtained by culling host 1 by the amount

$$
y=\frac{-0.468-0.132 a}{-0.04 a}+b=3.3+\frac{11.7}{a}+b
$$

Additional information is required to determine parameters $a$ and $b$ in equation (6).

\section{General Properties}

This "bottom-up" MSS culling strategy is just one of many ways of successfully culling a host community to exclude the pathogen. Whatever culling strategy is employed, there are some general observations we can make. First, all reservoir hosts have to be culled. If not, then we would end up (after culling) with a stable community that contained a reservoir host, which is in conflict with rule 1. Second, we may have to cull some non-reservoir hosts. This is clear from the third scenario in the two-host case and from the discussion of matrix (4c). Third, the non-reservoir hosts that are not culled will form a stable sub-community. If they do not, then we would contradict a rephrased rule 1: a stable (culled) community cannot contain within it an unstable sub-community. 
Rule 1 and other properties that we will use later follow from the Perron-Frobenius theorem (Cox and Miller 1970; Seneta 1973) when applied to the invasion matrix for an arbitrarily sized system with density-dependent transmission and no direct competition. This theorem is discussed in "Properties of Invasion Matrices" in the appendix. DeAngelis et al. (1979) and Hastings and Botsford (2006) have discussed the properties of similar matrices in solving particular ecological problems.

\section{Irreducibility}

For simplicity, we have until now supposed that the off-diagonal elements of the invasion matrix are all positive; in practice, however, direct transmission of the infection is significant only between a few pairs of hosts, and as such, many of these off-diagonal elements are effectively 0 (Dobson and Foufopoulos 2001). However, the results that we have obtained still apply, provided that the host community is irreducible, that is, that each host species can directly or indirectly infect all other host species. This property can easily be identified from the network graph describing the transmission of the infection within the community. This graph is constructed from the invasion matrix $\mathrm{J}$ by the following rule: there is a node for each of the $n$ hosts of the $n \times n$ matrix $\mathbf{J}$. If the $(i, j)$ element of $\mathbf{J}$ is nonzero, draw a directed (arrowed) arc from node $j$ to node $i$. The network graphs in figure $2 A, 2 C$, and $2 D$ are irreducible, whereas those in figure $2 B$ and $2 E$ are reducible (i.e., not irreducible). Host 2 is unable to infect host 1 in figure $2 B$, and host 1 is unable to infect either host 2 or host 3 in figure $2 E$. In figure $2 C$, host 1 cannot directly infect host 3 but can do so indirectly by first infecting host 2 . 
A:

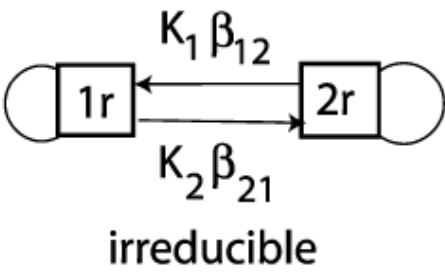

B:

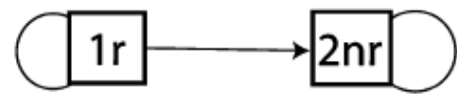

reducible

D:

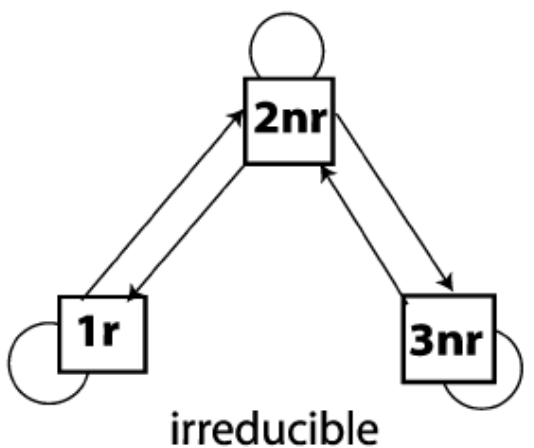

C:

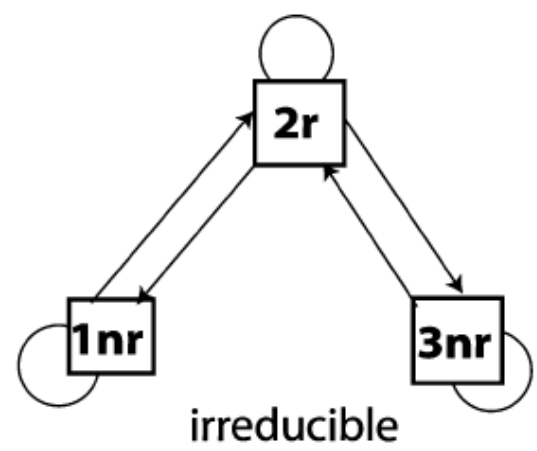

E:

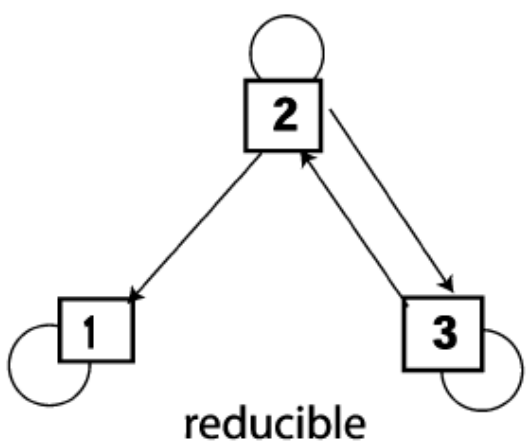

Figure 2: Irreducible $(A, C, D)$ and reducible $(B, E)$ infection transmission networks. The node integer indexes the host species, whereas $r$ denotes a reservoir host and $\mathrm{nr}$ denotes a non-reservoir host. Arrows on arcs indicate the direction of infection transmission; weights on arcs are the corresponding invasion matrix elements. See "Irreducibility" for discussion of culling strategies appropriate for the different network structures.

Figure $2 B$ models the Machupo virus epidemic, with rodents as the reservoir host ( $1 r$ ) and humans as the non-reservoir host $(2 \mathrm{nr})$ and with little or no transmission back to the reservoir host. Figure $2 A$ is a simple network model for bovine TB based on the SIS model (eq. [1]). It assumes that badgers (host 1 ) and cattle (host 2 ) are both reservoir hosts ( $r$ ) and as such can infect each other. Evidence for this comes from the work of Cox et al. (2005) and Woodroffe et al. (2006).

Figure $2 C$ and $2 D$ differs in the positioning of the single reservoir host $r$. In figure $2 C$, the single reservoir host lies in the "star" position (as host 2), in direct communication with the other (nonreservoir [nr]) hosts. One need only cull this star host, because the other hosts form a maximal stable community on their own. This observation generalizes to an arbitrarily sized system with (pairwise) direct transmission with only the star host. Vector transmission has a network with this structure, with the vector as the star host (Dobson and Foufopoulos 2001). If the reservoir host lies 
instead at one end of a chain of hosts (fig. 2D), then it may be that a second host has to be culled because hosts 2 and 3 together may support the pathogen.

For reducible networks, the invasion matrix $\mathbf{J}$ becomes block diagonal as far as the eigenvalues are concerned (Seneta 1973), and each block can be treated as a separate invasion matrix. For figure $2 E$, the exclusion of the pathogen from hosts 2 and 3 does not depend on the state of host 1 , and so the sub-community formed by host 2 and host 3 and the host 1 sub-community can be considered separately.

\section{Force of Infection}

Thus far we have looked for infection-control strategies that minimize the number of hosts that need to be culled. An alternative approach is to use the notion of "force of infection" as the basis for constructing the control strategy. To understand the issues involved, we need to refer to two further properties of the invasion matrix for an irreducible host community, which are highlighted in the Perron-Frobenius theorem. First, the leading eigenvalue I of the invasion matrix $\mathbf{J}$ (i.e., the eigenvalue with the largest real part) is in fact real and is positive when there is an invasion. If we cull all hosts by an amount greater than $\lambda$, then the leading eigenvalue becomes negative and the pathogen is excluded (because this culling reduces all the diagonal elements of $\mathbf{J}$ by the same amount and is equivalent to subtracting a multiple of the identity matrix from $\mathrm{J}$ ). The second relevant property of $\mathrm{J}$ is that $\lambda$ is bounded above and below

$$
\begin{aligned}
& c_{\text {min }} \leq \mathrm{I} \leq c_{\max },(7 \mathrm{a}) \\
& r_{\text {min }} \leq \mathrm{I} \leq r_{\max },(7 \mathrm{~b})
\end{aligned}
$$

where $c_{\max }$ is the maximum and $c_{\min }$ is the minimum of the column sums and $r_{\max }$ is the maximum and $r_{\min }$ is the minimum of the row sums. If $c_{\min }$ is positive, then the pathogen can invade (because then $\lambda$ is positive), but if $c_{\max }$ is negative, then the pathogen is excluded. If $c_{\max }$ and $c_{\min }$ are of opposite signs, then the sign of $\lambda$ is not determined by the constraints in equation (7a), and so more information is required. Similar arguments apply for the row sums. As an example, consider the following invasion matrices:

$$
\begin{aligned}
& \boldsymbol{J}=\left[\begin{array}{cc}
0.5 & 0.1 \\
0.3 & -0.4
\end{array}\right] \\
& \boldsymbol{J}=\left[\begin{array}{ccc}
-0.3 & 0.1 & 0.4 \\
0.1 & -0.2 & 0.5 \\
0.1 & 0.2 & 0.1
\end{array}\right] \\
& \boldsymbol{J}=\left[\begin{array}{cc}
A-d_{1} & B \\
C & D-d_{2}
\end{array}\right]
\end{aligned}
$$

For matrix (8a), the bounds are given by (i) $0.3 \leq \lambda \leq 0.8$ and (ii) $\_0.1 \leq \lambda \leq 1.2$. So the pathogen can invade because of inequalities (i), but inequalities (ii) are ambiguous on the issue.

The bounds in equations (7) suggest an alternative strategy for excluding the pathogen, that is, culling host species according to their column sums. Precisely, if the ith column sum is positive, then 
we cull the ith host by more than that column sum. The $(i, i)$ element, and hence the ith column sum, is reduced by the same amount. So, following this procedure, all column sums become negative and equation (7a) implies pathogen exclusion. The (non-reservoir) unculled hosts, with negative column sums, form a stable sub-community $S$ that will not, in general, be maximal because there might be non-reservoir hosts with positive column sums that would keep $S$ stable when they are added to $S$. For matrix (8a), both hosts are to be culled according to the top-down column-sum strategy, but only host 1 needs to be culled with the bottom-up MSS strategy. Our observations apply equally to row sums, and we note that only host 1 needs to be culled for the top-down row-sum strategy because the second row in matrix (8a) is negative. For matrix (8b), we need to only cull hosts 2 and 3 using column sums, we must cull all three hosts using row sums, and we need to cull only host 3 using the MSS strategy.

There is a third strategy, a mixed strategy, where we combine aspects of the top-down and bottomup strategies. We cull hosts in the order defined by their column or row sums, stopping when the remaining hosts form a stable sub-community $S 1$. For matrix (8b), host 3 (with the largest column sum) would be culled first, yielding the stable sub-community $S 1$ of hosts 1 and 2 . However, for the culled hosts, the minimum culling required is likely to be greater than the column or row sums if $S 1$ contains non-reservoir hosts with positive column or row sums, in order to compensate for their zero contribution to reducing the size of the infection reservoir. The bounds in equations (7a) and (7b) do not indicate by how much, however; this must be determined using a more detailed analysis. For example, according to equation (5), host 3 in matrix (8b) has to be culled by at least $x_{0}=$ $\operatorname{det} J / \operatorname{det} J_{3}=1.12$, where $J_{3}$ is the invasion matrix for the 3 sub-community formed by host 1 and host 2 . This is greater than the host 3 column sum, 1.0.

An alternative intuitive measure of the impact on host species of host $j$ being infected is the relative force of infection defined by $\sum K_{i} \beta_{i j} / d_{j}$ (summed over $i$; Dobson and Foufopoulos 2001). If we cull in the order dictated by this measure, we are progressively weakening the strongest agents of infection in the community. However, this strategy does not necessarily lead to the same culling strategy as that specified by the mathematical measure based on column or row sums, as matrix (8c) shows. With $A+C=18, B+D=15, d_{1}=7$, and $d_{2}=5$, the column sum criterion gives the ordering as host 1 and then host 2 , because $A+C-d_{1}>B+D-d_{2}$. For the relative force of infection measure, the culling order is reversed because $(A+C) / d_{1}<(B+D) / d_{2}$. This divergence in culling strategies arising from the duality between rows and columns and due to the different measures of influence is discussed further in "Optimization" in the appendix.

\section{Multiple Interactions}

\section{Direct Competition}

Consider a two-host community with direct competition that is modeled by replacing the growth rate $r_{i}\left(1-H_{i} / K_{i}\right)$ of model $(1)$ by $r_{1}\left(1-\frac{H_{i}}{K_{i}}-c_{i} H_{j}\right)$, where $i \neq j$. Direct competition decreases the susceptible equilibrium level $K_{i}$ by $\delta K_{i}$, where $k_{i}=\delta K_{i} / K_{i}$ is given by

$$
k_{i}=\frac{u_{i}\left(1-u_{j}\right)}{1-u_{i} u_{j}}
$$


where $i \neq j, u_{1}=c_{q} K_{2} / r_{1}$, and $u_{2}=c_{2} K_{1} / r_{2}$. Competition parameters $u_{1}$ and $u_{2}$ are considered to be less than 1 to ensure that direct competition does not exclude either host.

If host 2 is a non-reservoir host and is the much stronger competitor (i.e., $u_{2} \approx 0$ ), then the effect of introducing 2 host 2 to reservoir host 1 is to exclude the pathogen if the impact $u_{1}$ on host 1 is strong enough. Host 2 acts as if it is culling host 1, with culling rate $x$ given by equation (3) with equation (9) and $u_{2}=0$; that is, $x=u_{1} d_{1} /\left(1-u_{1}\right)$. Pathogen exclusion happens if $x \geq x_{0}$ (given by eq. [A2] in the appendix); that is, $u_{1}>1 /\left(1+d_{1} / x_{0}\right)$.

If host 1 has a non-negligible competitive impact on host 2, then the introduction of host 2 leads to a reduction in the carrying capacity of both hosts. The problem now is that the system may not be able to reach region $E$ for pathogen exclusion (fig. 1) because the constraints $u_{1}<1$ and $u_{2}<1$ for host survival limit the region of $(x, y)$ culling space that can be reached. Precisely, $(x, y)$ is limited by the constraint $x y \leq d_{1} d_{2}$ derived from equations (3) and (9). This happens in the following numerical example:

$$
K_{1} \beta_{11}=1.25, d_{1}=0.78, K_{1} \beta_{12}=0.2, K_{2} \beta_{21}=0.7, K_{2} \beta_{22}=0.85, d_{2}=0.05
$$

Here the pathogen cannot be excluded without excluding one of the host species as well. However, for a second example,

$$
K_{1} \beta_{11}=0.8, d_{1}=0.75, K_{1} \beta_{12}=0.1, K_{2} \beta_{21}=0.1, K_{2} \beta_{22}=0.6, d_{2}=0.5
$$

region $E$ can be reached for certain values of $u_{1}$ and $u_{2}$, for example, $u_{1}=0.3$ and $u_{2}=0.6$. The general situation is illustrated in figure $3 A$, where we super-impose the control boundary $C C$, $x y=d_{1} d_{2}$ on the threshold plot of figure $1 A$. The region $R$ of intersection in figure $3 A$ shows that the arrival of a second host can lead to the exclusion of the pathogen that is infecting the first host although each host is unable to resist infection when alone. This happens because, in bringing the two hosts together, two forces are activated: the force of infection that promotes pathogen persistence and the force of direct competition that reduces host abundance and thus discourages pathogen persistence.

\section{Frequency Dependence}

Suppose density-dependent infection transmission $(\beta I S)$ is replaced by frequency-dependent transmission $(\beta I S / H)$, where the transmission rate depends on the proportion (not the abundance) of infectious individuals in the total community (Hethcote et al. 2005). In modelling terms, this reformulation means replacing $K_{i}$ in the invasion matrix J with $K_{i} / \sum H$, where $\sum H$ denotes the sum of all the host populations (Rudolph and Antonovics 2005). For the SIS model, this sum reduces to the sum of the susceptible populations (at their carrying capacity levels) in the rare-invader approximation. As a consequence, the invasion matrix $\mathbf{J}$ depends only on the ratios of the carrying capacities. 

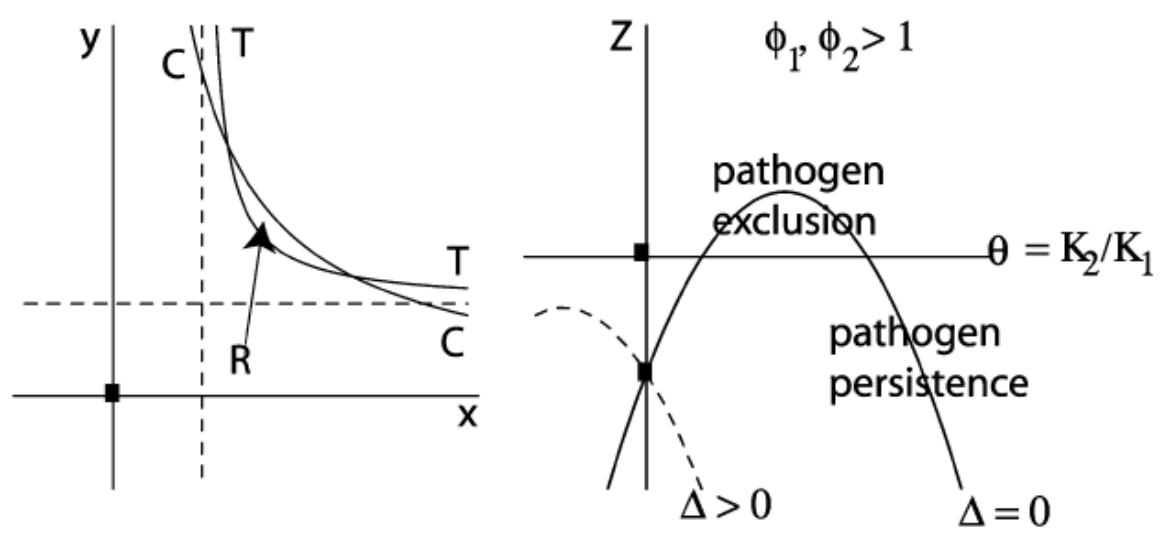

Figure 3: The phenomenon of two reservoir hosts excluding the pathogen with direct competition $(A)$ and frequency dependence $(B)$. In $A$, the control feasibility boundary $C C$ (i.e., the upper limit to control by direct competition without host exclusion) intersects the threshold $T$, and pathogen exclusion is possible in the intersection region $R . \ln B, \varphi_{i}=\beta_{i i} / d_{i}$ acts as basic reproduction numbers, $\Delta=\beta_{12} \beta_{21} / d_{1} d_{2}$ is a measure of cross-infection, and $\vee \mathrm{p} K / K$ is the control variable (the ratio of carrying capacities). $Z=(1+\theta)^{2} \operatorname{det} J / d_{1} d_{2}$, where $\mathrm{J}$ is the invasion matrix. $B$ shows the range of $v$ and $D$, where pathogen exclusion is possible when both hosts satisfy the restriction $\varphi_{i}=\beta_{i i} / d_{i}>1$.

Consider the case of two host species where intra-transmission rates are sufficiently high such that $\varphi_{i}=\beta_{i i} / d_{i}$ (where $i=1,2$ ). These indices act as basic re-production numbers, and we will refer to such hosts as reservoir hosts (see McCallum et al. 2001). If cross-infection ( $\left.\Delta=\beta_{12} \beta_{21} / d_{1} d_{2}\right)$ is sufficiently low and carrying capacities are not too different, then together these two hosts will exclude the pathogen. This is shown in figure $3 B$, where we plot $Z$ (proportional to the determinant of the invasion matrix) against the control parameter $\theta=K_{2} / K_{1}$ (see "Generalized Culling" in the appendix for details). Thus, with frequency dependence it is possible to exclude a pathogen from a host by adding another (not directly competing) reservoir host. This is the phenomenon we observed with direct competition. For frequency dependence, there is an implicit second interaction, with the effective infection transmission rate within a species dependent on the states of the other species (see again "Generalized Culling" in the appendix).

Frequency dependence has no impact on vaccination because, with vaccination, the total population $H_{i}$ is not reduced. It shifts individuals between compartments, from susceptible to immune, keeping $H_{i}, \sum H$, and the frequency-dependent denominator constant. The invasion matrix depends on the carrying capacity levels, not just on their ratio.

\section{Discussion}


The first and often the most difficult task in eradicating a multi-host infection is determining the key reservoir hosts (Johnson et al. 1965; Cleaveland and Dye 1995; Haydon et al. 2002). When these are known, one must use models to test possible control strategies before they are implemented. Our purpose has been to contribute to an understanding of how one might be able to exploit the structure of epidemiological models to gain control over the pathogen.

An exploration of the structure of the multi-host SIS model (eq. [1]), generalized to describe an arbitrary number of hosts, has led to the following observations that are of relevance in designing a culling strategy. All reservoir hosts need to be culled. In general, some non-reservoir hosts also need to be culled but not those with a negative column or row sum. The set of hosts with negative column or row sums and the set of unculled hosts both form sub-communities that are stable against pathogen invasion. Our proposed hybrid culling strategy, where we cull in order of decreasing column or row sums until a stable sub-community is encountered, combines the stable and unstable nesting properties of host sub-communities and the column or row sums as indicators of community instability.

We have been able to derive these general results by focusing on the threshold properties of the system, as described by the pathogen invasion matrix. This matrix can easily be transformed into a nonnegative matrix, a class of matrices whose properties have been extensively studied elsewhere (Seneta 1973). We thereby avoid the difficult task of analysing the stability properties of equilibria where the pathogen coexists with its hosts. The rare-invader approximation, which we have used, separates the analysis into simpler tasks: first, an equilibrium analysis in the absence of infection and second, an invasion analysis based on the results of this equilibrium analysis.

When extra structure is added to the SIS model, the threshold analysis can still apply. This is the case with direct competition, predation, and frequency dependence. The mathematics stays the same, with the invasion matrix related to a nonnegative matrix, but the biological interpretation requires clarification. When we say "when alone," as we have throughout this section, we now mean "when alone with respect to infection but not with respect to other forces present." In equation (10), neither host supports the pathogen at the susceptible population level when direct competition is present, but both do so if separation entails cutting both apparent and direct competition links. Latency can also be included within our methodology, as discussed in "Threshold Analysis of the Two-Host Susceptible-Exposed-Infectious-Recovered (SEIR) Model" in the appendix. We are currently performing detailed analyses of systems with added structure, and we hope to publish our findings in due course.

In practice, the final choice of control strategy is determined by a wide range of considerationsecological, physical, regulatory, and economic-that are specific to the situation being considered. To illustrate this point, consider again our simple model of bovine TB, based on equation (1), with the network structure of figure $2 A$. In this model, it is assumed that both badgers and cattle are reservoir hosts infecting each other. According to our theory, the pathogen cannot be excluded just by testing and culling the cattle population; the badger population must be culled as well (King 2007). However, the degree of culling required may not be consistent with conservation objectives or cost constraints (Bourne et al. 2007). For cattle, culling is limited by the efficiency of the testing procedures and, because of the cost involved, the frequency of testing (Cox et al. 2005). Subject to these qualifications, this simple model might provide a reasonable approximation when culling is 
proactive and done over a large area (Donnelly et al. 2006), but it is unlikely to do so with reactive culling where social disruption of badger communities can lead to an increase in bovine TB (Donnelly et al. 2003), an interesting example of compensatory mortality. This feedback mechanism can be modelled by adding more nodes to the network in figure $2 A$ to describe different badger populations linked together by migration and infection transmission arising from increased ranging. We are currently investigating this model.

Our general threshold methodology, applicable to a wide range of epidemiological models, provides valuable insights into how to design a suitable culling strategy to exclude a pathogen. It identifies which constraints may apply and which approaches or combination of approaches might best fit the ecological system being studied.

\section{Acknowledgments}

We would like to thank our colleagues in the Mathematics Group and the School of Biological and Environmental Sciences at the University of Stirling, in particular B. Mestel and R. Norman for their useful discussions. We also appreciate the constructive comments of two anonymous reviewers. The numerical and algebraic calculations in our study were performed using an Apple Macintosh G4 computer and the software package Mathematica.

\section{Literature Cited}

Anderson, R. C. 1972. The ecological relationship of meningeal worms and native cervids in North America. Journal of Wildlife Diseases 8:304-310.

Anderson, R. M., and R. M. May. 1986. The invasion, persistence and spread of infectious diseases within animal and plant communities. Philosophical Transactions of the Royal Society B: Biological Sciences 314:533-573.

Bourne, J., C. A. Donnelly, D. R. Cox, G. Gettinby, J. P. Mclnerney, W. I. Morrison, and R. Woodroffe. 2007. Response to "Tuberculosis in cattle and badgers: a report by the chief scientific officer." http://www.defra.gov.uk/animalh/tb/isg/pdf/isg-responsetosirdking.pdf

Cleaveland, S., and C. Dye. 1995. Maintenance of a micro-parasite infecting several host species: rabies in the Serengeti. Parasitology 111:S33-S47.

Cox, D. R., and H. D. Miller. 1970. The theory of stochastic processes. Methuen, London.

Cox, D. R., C. A. Donnelly, F. J. Bourne, G. Gettinby, J. P.McInerney, W. I. Morrison, and R. Woodroffe. 2005. Simple model for tuberculosis in cattle and badgers. Proceedings of the National Academy of Sciences of the USA 102:17588-17593.

Daszak, P., A. A. Cunningham, and A. D. Hyatt. 2000. Emerging diseases of wildlife: threats to biodiversity and human health. Science 287:443-449.

DeAngelis, D. L., C. C. Travis, and W. M. Post. 1979. Persistence and stability of seed-dispersed species in a patchy environment. Theoretical Population Biology 16:107-125. 
Dobson, A. 2004. Population dynamics of pathogens with multiple host species. American Naturalist 164(suppl):S64-S78.

Dobson, A., and J. Foufopoulos. 2001. Emerging infectious pathogens of wildlife. Philosophical Transactions of the Royal Society B: Biological Sciences 356:1001-1012.

Donnelly, C. A., R. Woodroffe, D. R. Cox, J. Bourne, G. Gettinby, A. M. LeFevre, J. P. Mclnerney, and W. I. Morrison. 2003. Impact of localized badger culling on tuberculosis incidence in British cattle. Nature 426:834-837.

Donnelly, C. A., R. Woodroffe, D. R. Cox, F. J. Bourne, C. L. Cheeseman, R. S. Clifton-Hadley, G.Wei, et al. 2006. Positive and negative effects of widespread badger culling on tuberculosis in cattle. Nature 439:843-846.

Ferguson, N. M., C. A. Donnelly, and R. M. Anderson. 2001. Transmission intensity and impact of control policies on the foot and mouth epidemic in Great Britain. Nature 413:542-547.

Garrett, L. 1994. The coming plague. Penguin, New York.

Hastings, A., and L. W. Botsford. 2006. Persistence of spatial populations depends on returning home. Proceedings of the National Academy of Sciences of the USA 103:6067-6072.

Haydon, D. T., S. Cleaveland, L. H. Taylor, and M. K. Laurenson. 2002. Identifying reservoirs of infection: a conceptual and practical challenge. Emerging Infectious Diseases 8:1468-1473.

Hethcote, H. W., W. Wang, and Y. Li. 2005. Species coexistence and periodicity in host-hostpathogen models. Journal of Mathematical Biology 51:629-660.

Holt, R. D., and J. Pickering. 1985. Infectious disease and species coexistence: a model in LotkaVolterra form. American Naturalist 126:196-211.

Hudson, P., and J. Greenman. 1998. Competition mediated by parasites: biological and theoretical progress. Trends in Ecology \& Evolution 13:387-390.

Johnson, K. M., N. Wiebenga, and R. Mackenzie. 1965. Virus isolations from human cases of hemorrhagic fever in Bolivia. Proceedings of the Society of Experimental Biology and Medicine 118:113-118.

King, D. 2007. Bovine tuberculosis in cattle and badgers: a report by the chief scientific adviser, Sir David King. http://www.defra.gov.uk/animalh/tb/pdf/badgersreport-king.pdf.

Krebs, J. R., R. M. Anderson, T. Clutton-Brock, C. A. Donnelly, S. Frost, W. I. Morrison, R. Woodroffe, and D. Young. 1998. Badgers and bovine TB: conflicts between conservation and health. Science 279:817-818.

McCallum, H., N. Barlow, and J. Hone. 2001. How should pathogen transmission be modelled? Trends in Ecology \& Evolution 16:295-300.

McLean, A., R. May, J. Pattison, and R. Weiss. 2006. SARS: a case study in emerging infections, Oxford University Press, Oxford. 
Petersen, L. R., and J. T. Roehrig. 2001.West Nile virus: a re-emerging global pathogen. Emerging Infectious Diseases 7:611-614.

Roberts, M. G. 1996. The dynamics of bovine tuberculosis in possum populations and its eradication by culling or vaccination. Journal of Animal Ecology 65:451-464.

Rudolph, V. H. W., and J. Antonovics. 2005. Species coexistence and pathogens with frequencydependent transmission. American Naturalist 166:112-118.

Seneta, E. 1973. Non-negative matrices. Allen \& Unwin, London.

Swinton, J., J. Harwood, B. T. Grenfell, and C. A. Gilligan. 1998. Persistence thresholds for phocine distemper virus infection in harbour seal Phoca vitulina metapopulations. Journal of Animal Ecology 67:54-68.

Tompkins, D. M., J. V. Greenman, and P. J. Hudson. 2001.Differential impact of a shared nematode parasite on two gamebird hosts: implications for apparent competition. Parasitology 122:187-193.

Woodroffe, R., C. A. Donnelly, H. E. Jenkins, W. T. Johnston, D. R. Cox, F. J. Bourne, C. L. Cheeseman, et al. 2006. Culling and cattle controls influence tuberculosis risk for badgers. Proceedings of the National Academy of Sciences of the USA 103:14713-14717. 